BMJ Open

Diabetes

Research

\& Care

\section{Role of depression in diabetes management in an ethnic minority population: a case of Korean Americans with type 2 diabetes}

To cite: Kim MT, Kim KB, Ko J, et al. Role of depression in diabetes management in an ethnic minority population: a case of Korean Americans with type 2 diabetes. BMJ Open Diabetes Research and Care 2017;5:e000337. doi:10.1136/bmjdrc-2016000337

Received 3 October 2016 Revised 12 January 2017 Accepted 29 January 2017

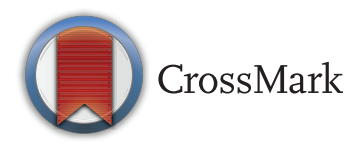

${ }^{1}$ School of Nursing, University of Texas at Austin, Austin, Texas, USA

${ }^{2}$ Korean Resource Center, Ellicott City, Maryland, USA ${ }^{3}$ School of Social Work, University of Texas at Austin, Austin, Texas, USA

${ }^{4}$ School of Medicine, Johns Hopkins University, Baltimore, Maryland, USA ${ }^{5}$ School of Medicine, Yale University, Boston, Connecticut, USA

Correspondence to Dr Kim Byeng Kim; kbkim@ikorean.org

\author{
Miyong To Kim, ${ }^{1}$ Kim Byeng Kim, ${ }^{2}$ Jisook Ko, ${ }^{1}$ Yuri Jang, ${ }^{3}$ David Levine, ${ }^{4}$ \\ Hochang Benjamin Lee ${ }^{5}$
}

ABSTRACT

Background: Comorbid depression and diabetes mellitus (DM) compound challenges to disease management such as low health literacy, insufficient access to care, and social or linguistic isolation. Korean Americans (KAs), predominantly first-generation immigrants, suffer from a high prevalence of type 2 DM and depression. Limited research on KAs has prevented the development of effective interventions. Objectives: To compare the prevalence of depression in KAs with DM and all Americans with/without DM, and to explore correlates of comorbid DM and depression and strategies to address KAs' DM and depression.

Methods: KAs' data were from a clinical trial of a community-based self-help intervention to improve KAs' DM and mental health outcomes. National Health and Nutrition Examination Survey data sets enabled comparison. Clinical indicators included hemoglobin A1C, lipid panel, and body mass index.

Psychobehavioral indicators included self-efficacy for DM management, quality of life, and depression (Patient Health Questionnaire-9 (PHQ-9)).

Results: More KAs with DM had depression (44.2\%) than did all Americans with DM (28.7\%) or without DM (20.1\%). Significantly more KAs with DM had mild $(29.3 \%)$ or clinical $(14.9 \%)$ depression than did Americans with DM (mild, $17.2 \%$; clinical, $11.5 \%$ ) or without (mild, $13.8 \%$; clinical, $6.3 \%$ ). One of six KAs with DM (16.9\%) thought of suicide or self-harm (Americans with/without $=5.0 \%, 2.8 \%$ ). The self-help intervention reduced the mean $\mathrm{PHQ}-9$ from 5.4 at baseline to 4.1 at 12 months.

Limitations: External validity might be limited; KAs' data were from one study site.

Conclusions: The prevalence of depression and DM among KAs warrants the development of efficacious interventions.

Trial registration number: NCT01264796.

\section{INTRODUCTION}

Depression and diabetes mellitus (DM) are the most common comorbid conditions across all sociodemographic spectra. ${ }^{1}$

\section{Significance of this study}

What is already known about this subject? Depression is a significant comorbid risk for diabetes.

\section{What are the new findings?}

Korean Americans with type 2 diabetes mellitus have a high level of depression prevalence $(44.2 \%)$.

How might these results change the focus of research or clinical practice?

A community-based self-help programme is effective in mitigating depression.

Although their etiological relationship is unclear, together they present significant challenges to disease management in individuals with type $2 \mathrm{DM}^{2-4}$ In comparison with people who have DM but no depression, individuals with concurrent depression and DM report lower levels of self-efficacy, selfcare, and quality of life and have poorer clinical outcomes, including higher incidences of complications and reduced life expectancy. ${ }^{5}$ Comorbid depression and DM increase treatment costs, and clinical outcomes have not yet demonstrated their treatments' effectiveness. ${ }^{6-9}$ Also, the difficulty of DM management is amplified in vulnerable subgroups: individuals with disabilities, low income, and low health literacy, as well as members of linguistically isolated ethnic minority immigrant groups.

Korean Americans (KAs) represent such a group. KAs, predominantly first-generation immigrants, suffer from a disproportionally high incidence of depression ${ }^{10} 11$ and $\mathrm{DM},{ }^{12}$ and they are socially isolated by linguistic and cultural barriers. A recent epidemiological study of elderly KAs (age $\geq 60$ ) has reported a prevalence of depression of over $30 \%$ on the Patient Health Questionnaire (PHQ-9; mild depression score, 5-9; clinical 
depression, $\geq 10) ;{ }^{13} \mathrm{DM}$ was significantly correlated with depression. As depression affects individuals' abilities at DM self-management as well as clinical outcomes, future DM treatment and interventions will require a holistic approach, integrating strategies to improve mental and physiological health. Such intervention requires a thorough understanding of the target population, including the prevalence of factors that contribute to their chronic conditions as well as unique contextual factors that may influence their disease management. Such critical information, however, is not yet available due to the lack of empirical studies of KAs with type 2 DM that characterize the role of depression in their disease management.

For the present study, we have analyzed data from a recently completed clinical trial of a community-based DM self-management intervention by estimating the prevalence of depression in the ethnic minority population of KAs and examining both the role of depression in their DM self-management behaviors and their clinical outcomes. To make clear inferences, we have also used national data from the National Health and Nutrition Examination Survey (NHANES) for the years 2005-2006, 2007-2008, 2009-2010, and 2011-2012 as a reference group. In addition to explicating empirical relationships between the comorbid conditions of DM and depression and traditional factors that influence DM selfmanagement and clinical outcomes, we have evaluated several unique contextual factors associated with the KA population. In undertaking this study, we hypothesized that KAs with DM would have a higher prevalence of depression than would the general population and that this would have a significant impact on their ability to manage DM and thus on their quality of life.

\section{MATERIAL AND METHODS \\ Design}

The primary data source for this study was an open-label randomized control trial to test the effectiveness of a community-based self-help intervention for KAs with uncontrolled $\mathrm{DM}$ as measured by hemoglobin $\mathrm{A} 1 \mathrm{C}$ $\geq 7.0 \%$. A total of $250 \mathrm{KAs}$ residing in the BaltimoreWashington region enrolled in the study. After 12 months, 105 in the intervention group $(n=120)$ and 104 in the control group $(n=130)$ completed the program for a retention rate of $83.6 \%$. Recruitment, enrollment, and retention of participants have been detailed elsewhere. ${ }^{1415}$ The study protocol was approved by the Johns Hopkins Institutional Review Board. After providing their consent, participants enrolled in the study from September 2009 to June 2014.

\section{Intervention}

The intervention group received 2 hours of didactic training each week for 6 weeks, followed by monthly telephone counseling by a team of registered nurses and community health workers. The control group received a shortened version of the intervention after the study was completed. The intervention's details have been presented elsewhere. ${ }^{14}{ }^{15}$ In short, the training covered participants' DM management and control. It included the etiology of DM, healthy diet, and exercise. One hour devoted to stress management included ways to improve communication and solve problems.

\section{Measurements}

Our clinical trial and the NHANES followed the same data collection protocol and included (a) demographics, (b) dietary data, (c) examination data, (d) laboratory data, and (e) questionnaire data. Our data sets, however, were on a smaller scale. Whereas the NHANES data are biannual and cross-sectional, ours were panel data, collected at baseline and at 3, 6, 9, and 12 months, except for the dietary data and the questionnaire data for depression, which were not collected at 9 months.

Demographic information included age, sex, marital status, family size, work status, and annual household income. Family size was an indicator of living arrangement, recoded as 1 for living alone or 0 for otherwise. In our study, participants reported actual monthly income, which was converted to a percentage of the federal poverty line by taking the family size into account. It was further reclassified into four categories, according to the Patient Protection and Affordable Care Act: 0 for an income $\leq 138 \%$; 1 for $139-250 \%$; 2 for $251-400 \%$, and 3 for $>400 \%$. Dietary data were collected using the 24-hour recall method. Examination data included weight and height for the calculation of body mass index (BMI), as well as blood pressure measurement. Laboratory data included hemoglobin A1C, lipid panel (total cholesterol, triglycerides), and fasting plasma glucose.

The PHQ-9 measured depression. The PHQ's 9 items probe the frequency of depressive experience-for example, 'Over the past 2 weeks, how often have you been bothered by any of the following problems?' Responses are 0='Not at all', 1='Several days', 2='More than half the days', and $3=$ 'Nearly every day'. Thus, the total score ranges from 0 to 27 . In our trial, we used the instrument's Korean version, the PHQ-9K. ${ }^{16}{ }^{17}$ The total score was reclassified into three ordered categories of depression: (a) 'normal' if the score was $<5$, (b) 'mild depression' if the score was 5-9, and (c) 'clinical depression' if the score was 10 or higher. ${ }^{16}$ In addition, for detailed analysis, each item score was recoded as 0 for 'no depression episode' or 1 for 'episode'. Also included as two dichotomous indicators were DM medication and whether or not an individual was on insulin ('yes' $=1$ and 'no' $=0$ ).

Self-efficacy measured the level of confidence in managing DM with regard to diet, exercise, and general selfmanagement behaviors, using a DM-related self-efficacy scale $^{18}$ with eight 10 -point Likert-type items (1='None', and $10=$ 'Full'), yielding a total sum from 8 to 80 . Social support from family members and friends was measured with the Personal Resource Questionnaire, ${ }^{19}{ }^{20}$ with 11 
5-point Likert-type items (1='Receiving no support', and $5=$ "A lot of support'). We also scored the gap between expected and received support on the same questionnaire. Total scores for self-efficacy, social support, and support gap were reclassified using tertiles: $0=$ 'Low', 1='Middle', and 2='High'. The self-efficacy and the support scales were available only in our clinical study.

DM control was measured by hemoglobin A1C, the proportion of glycated red cells (expressed in percentages). A1C is a gold standard for the diagnosis of DM and indication of DM control. ${ }^{21} \mathrm{~A}$ person has DM if $\mathrm{A} 1 \mathrm{C} \geq 6.5 \%$, and pre-diabetes if $\mathrm{A} 1 \mathrm{C}$ is $5.7-6.4 \%$.

\section{Analysis}

Depression prevalence among KAs with DM is based on our data, excluding one incomplete observation. For comparison, we selected two additional groups from the NHANES 2005-2006, 2007-2008, 2009-2010, and 20112012 cycles. One, the comparison group, included Americans (whites, blacks, Mexican Americans, Hispanics, and others, $\mathrm{n}=1707$ ) with uncontrolled DM (A1C $\geq 7.0 \%$ ); the other, the standard group, consisted of Americans $(\mathrm{n}=6880)$ without DM, pre-diabetes, or type $1 \mathrm{DM}$ (A1C <5.7\%). To explicate correlates of depression, ordered logistic regression was administered on a pooled sample of KAs at baseline, 3, 6, and 12 months ( $\mathrm{n}=759$ with listwise deletion), on the comparison and standard groups. Further, to explore the self-help intervention's effects on depression among KAs with uncontrolled DM, a mixed effects model was used on our panel data. We used STATA (V.14) for analysis; statistical significance was set at $\mathrm{p}<0.05$.

\section{RESULTS}

\section{Sample characteristics}

The KAs from the clinical trial $(\mathrm{N}=249)$ were similar to the comparison group (Americans with $\mathrm{DM}, \mathrm{n}=1707$ ) in mean $( \pm \mathrm{SE})$ age $(58.3 \pm 0.5$ vs $58.4 \pm 0.4$ years $)$. More men were included in the KA sample $(56.6 \%)$ than in the comparison group $(51.0 \%)$. More KAs were working either full or part-time $(59.5 \%$ vs $44.8 \%)$, with greater education $(86.3 \%$ vs $71.5 \%$ for high school graduate or above). More KAs reported low income than did the comparison group $(67.1 \%$ vs $53.9 \%$ making less $251 \%$ of the federal poverty line). The majority of KAs were married $(89.6 \%$ vs $59.0 \%)$, and fewer KAs were living alone $(6.0 \%$ vs $16.1 \%)$ or covered by health insurance (52.2\% vs $84.3 \%)$.

Regarding physiological indicators, the mean (SE) BMI of KAs was $25.4(0.2)$, lower than that of the comparison group (mean=32.2, $\mathrm{SE}=0.2$ ). Although KAs had the same level of total cholesterol $(193 \mathrm{mg} / \mathrm{dL})$ as did Americans with DM, they had lower triglycerides (191 vs $240 \mathrm{mg}$ / $\mathrm{dL}$ ) and fasting serum glucose (160 vs $188 \mathrm{mg} / \mathrm{dL})$. Also, more KAs were taking DM medication $(63.1 \%$ vs $47.4 \%$, unweighted $\mathrm{z}=4.63,95 \%$ CI 0.09 to $0.22, \mathrm{p}<0.001$ ), but significantly fewer KAs were receiving insulin injection therapy $(4.2 \%$ vs $23.2 \%$; unweighted $\mathrm{z}=-6.90,95 \%$ CI -0.22 to $-0.16, \mathrm{p}<0.001$ ) (see table 1 ).

\section{Prevalence of depression}

Overall, more KAs reported depression than did those in the comparison and standard groups. Differences were statistically significant for all items except item 8 , 'Moving or speaking so slowly that other people could have noticed...' (unweighted $\mathrm{z}=1.43,95 \%$ CI -0.02 to $0.08, \mathrm{p}=0.153$ ). In particular, about twice as many KAs than those in the comparison group reported 'Little interest or pleasure in doing things' (49.8\% vs 27.7\%; unweighted $\mathrm{z}=7.1,95 \%$ CI 0.16 to $0.29, \mathrm{p}<0.001$ ), 'Poor appetite or overeating' $(55.4 \%$ vs $26.4 \%$; $\mathrm{z}=9.3,95 \% \mathrm{CI}$ 0.22 to $0.36, \mathrm{p}<0.001$ ), and 'Trouble concentrating on things, such as reading the newspaper or watching television' $(29.3 \%$ vs $15.9 \% ; \mathrm{z}=5.2,95 \%$ CI 0.07 to 0.19 , $\mathrm{p}<0.001)$.

The mean $( \pm \mathrm{SE})$ depression scores of KAs $(5.1 \pm 0.3)$ and Hispanics (5.0 \pm 0.7$)$ were highest among races; differences between KAs and the comparison $(3.7 \pm 0.1)$ and standard $(2.7 \pm 0.1)$ groups were statistically significant (unweighted $\mathrm{t}=4.12$ and 7.12 , respectively, $\mathrm{p}<0.001$ ). Accordingly, about a third $(29.6 \%)$ of KAs had mild depression, which was higher than in whites $(16.9 \%)$, blacks (16.6\%), Mexican Americans (18.0\%), Hispanics $(20.1 \%)$, and others $(17.5 \%)$. Similarly, clinical depression among KAs (14.9\%) was second highest after clinical depression in Hispanics $(19.7 \%)$ followed by whites $(11.5 \%)$, blacks $(11.2 \%)$, and Mexican Americans $(11.0 \%)$. As a result, depression (mild and clinical depression combined) was most prevalent among KAs $(44.2 \%)$, who were followed closely by Hispanics $(39.8 \%)$ and other races with margins of $>15$ percentage points.

About one out of six KAs $(16.9 \%)$ had thoughts of hurting or killing themselves within the past 2 weeks, which was $>3$ times the percentage for other ethnic groups $(4.8 \%$ for whites, $5.4 \%$ for blacks, and $5.5 \%$ for Mexican Americans and others). The KAs' rate on this item was 5.8 times higher than that for the standard group $(2.9 \%)$. Nevertheless, KAs underestimated the impact of depression on daily activities (eg, doing one's work, taking care of things at home, or getting along with other people). One of four KAs $(24.4 \%)$ felt impacts of depression, which was significantly lower than in the comparison group $(33.6 \%)$ (unweighted $\mathrm{z}=-2.90,95 \%$ CI -0.15 to $-0.03, \mathrm{p}=0.004$ ), but similar to impacts of depression in the standard group (26.6\%) (see table 2).

\section{Correlates of depression}

Ordered logistic regression analysis was performed to identify correlates of depression on KAs from our clinical trial, the comparison group, and the standard group.

First, several demographic and physiological characteristics were correlated with the incidence of depression for KAs as well as other Americans, although the 
Table 1 Comparison of demographic and physiological indicators between the Korean Americans ( $\geq 35$ years old) with uncontrolled type 2 diabetes (A1C $\geq 7.0 \%$ ) at baseline in our clinical study, the comparison group (Americans with the same conditions as Korean Americans), and the standard group (Americans with A1C $<5.7 \%$ and not being diagnosed of diabetes,

pre-diabetes or type 1 diabetes) in the NHANES

\begin{tabular}{|c|c|c|c|c|c|c|c|c|}
\hline \multirow[b]{2}{*}{ Indicatorslgroups } & \multirow[b]{2}{*}{$\begin{array}{l}\text { Korean Americans* } \\
(n=249)\end{array}$} & \multicolumn{6}{|c|}{ Comparison group $\dagger$} & \multirow[b]{2}{*}{$\begin{array}{l}\text { Standard } \\
\text { group } \neq(n=6880)\end{array}$} \\
\hline & & $\begin{array}{l}\text { Total } \\
(n=1707)\end{array}$ & $\begin{array}{l}\text { Whites } \\
(n=537)\end{array}$ & $\begin{array}{l}\text { Blacks } \\
(n=574)\end{array}$ & $\begin{array}{l}\text { Mexican Americans } \\
(\mathrm{n}=314)\end{array}$ & $\begin{array}{l}\text { Hispanic } \\
(n=161)\end{array}$ & $\begin{array}{l}\text { Others } \\
(n=121)\end{array}$ & \\
\hline Male (\%) & 56.6 & 51.0 & 53.2 & 45.5 & 54.3 & 46.1 & 49.5 & 48.1 \\
\hline Age, mean (SE) (years) & $58.3(0.5)$ & $58.4(0.4)$ & $60.5(0.6)$ & $56.7(0.6)$ & $54.5(1.0)$ & $52.7(1.5)$ & $57.2(1.6)$ & $51.6(0.3)$ \\
\hline Married (\%) & 89.6 & 59.0 & .62 .6 & .44 .7 & 59.6 & 56.8 & 73.7 & 65.4 \\
\hline Living alone (\%) & 6.0 & 16.1 & 18.6 & 18.5 & 9.1 & 7.0 & 7.2 & 14.1 \\
\hline Working full/part-time (\%) & 59.5 & 44.8 & 43.1 & 44.9 & 50.1 & 39.0 & 55.4 & 68.0 \\
\hline \multicolumn{9}{|l|}{ Education } \\
\hline$<$ High school graduate (\%) & 13.7 & 28.5 & 18.9 & 32.1 & 70.2 & 51.1 & 18.5 & 14.2 \\
\hline High school graduate $(\%)$ & 36.1 & 25.8 & 30.1 & 25.8 & 13.1 & 13.2 & 19.6 & 22.0 \\
\hline$\geq$ College graduate $(\%)$ & 50.2 & 45.7 & 51.0 & 42.1 & 16.7 & 35.8 & 62.0 & 63.8 \\
\hline \multicolumn{9}{|l|}{ Income (federal poverty line) } \\
\hline$\leq 138 \%$ & 38.0 & 29.8 & 23.0 & 31.0 & 55.1 & 51.6 & 29.2 & 16.4 \\
\hline$\leq 250 \%$ & 29.1 & 24.1 & 21.7 & 28.0 & 28.8 & 24.0 & 26.4 & 17.1 \\
\hline$\leq 400 \%$ & 17.4 & 18.8 & 21.3 & 22.5 & 7.1 & 12.6 & 9.8 & 21.3 \\
\hline$>400 \%$ & 15.5 & 27.3 & 34.0 & 18.5 & 9.0 & 11.8 & 34.6 & 45.2 \\
\hline Health insurance (\%) & 52.2 & 84.3 & 90.9 & 84.1 & 55.3 & 73.8 & 81.1 & 85.4 \\
\hline BMI, mean (SE) & $25.4(0.2)$ & $32.2(0.3)$ & $32.8(0.5)$ & $32.9(0.4)$ & $31.5(0.5)$ & $31.7(0.6)$ & $28.6(1.2)$ & $27.8(0.1)$ \\
\hline Normal (BMI <25) & 47.4 & 16.1 & 14.6 & 14.6 & 12.7 & 11.8 & 39.5 & 32.3 \\
\hline Overweight $(\mathrm{BMI}<30)$ & 45.8 & 24.2 & 21.4 & 25.8 & 32.6 & 30.9 & 23.6 & 38.1 \\
\hline Obese $(\mathrm{BMI} \geq 30)$ & 6.8 & 59.7 & 64.0 & 59.6 & 54.7 & 57.3 & 36.9 & 28.6 \\
\hline A1C (\%), mean (SE) & $8.8(0.1)$ & $8.6(0.1)$ & $8.3(0.1)$ & $8.9(0.1)$ & $9.1(0.1)$ & $9.1(0.3)$ & $8.7(0.2)$ & $5.3(0.0)$ \\
\hline Total cholesterol, mean (SE) & $193(2.9)$ & $193(1.7)$ & $187(2.4)$ & $196(3.0)$ & $206(3.5)$ & $206(6.5)$ & $193(7.6)$ & $205(0.8)$ \\
\hline Normal $(<200)$ & 59.6 & 59.4 & 59.6 & 65.6 & 55.0 & 45.5 & 40.3 & 47.3 \\
\hline Borderline (200-239) & 26.4 & 26.5 & 24.2 & 20.5 & 30.1 & 29.9 & 35.4 & 35.8 \\
\hline High $(\geq 240)$ & 14.0 & 14.1 & 16.2 & 13.9 & 14.9 & 24.6 & 24.3 & 16.9 \\
\hline Triglyceride status (mg/dL) & $191(12.8)$ & $240(8.5)$ & $250(11.4)$ & $170(7.2)$ & $276(22.3)$ & $265(26.0)$ & $252(29.7)$ & $151(2.0)$ \\
\hline Normal $(<150)$ & 50.6 & 37.8 & 35.2 & 56.1 & 31.7 & 33.7 & 27.0 & 64.3 \\
\hline Borderline (150-199) & 22.5 & 16.9 & 17.6 & 17.1 & 18.1 & 11.9 & 14.5 & 15.5 \\
\hline High $(\geq 200)$ & 26.9 & 45.3 & 47.2 & 26.8 & 50.2 & 54.4 & 58.5 & 21.2 \\
\hline Blood pressure, SBP/DBP $(\mathrm{mg} / \mathrm{Hg})$ & $133 / 78$ & $130 / 71$ & $131 / 70$ & $134 / 73$ & $129 / 71$ & $127 / 73$ & $126 / 72$ & $121 / 72$ \\
\hline$<120 / 80$ & 37.3 & 29.8 & 30.0 & 25.2 & 31.4 & 36.4 & 33.1 & 45.8 \\
\hline$\leq 139 / 89$ & 39.4 & 46.1 & 44.5 & 48.1 & 49.4 & 47.1 & 48.8 & 44.2 \\
\hline$\geq 140 / 90$ & 23.3 & 24.1 & 25.5 & 26.7 & 19.2 & 16.5 & 18.1 & 10.0 \\
\hline Blood glucose (mg/dL) & $160(3.8)$ & $188(2.9)$ & $181(3.9)$ & $193(6.5)$ & $212(6.9)$ & $202(11.5)$ & $176(7.5)$ & $92(0.2)$ \\
\hline Normal $(<100)(\%)$ & 9.2 & 9.2 & 08.8 & 14.2 & 04.5 & 09.1 & 8.4 & 79.2 \\
\hline Pre-diabetes $(\leq 125)(\%)$ & 23.3 & 11.7 & 12.0 & 09.5 & 10.2 & 18.5 & 10.4 & 18.5 \\
\hline Diabetes $(>125)(\%)$ & 67.5 & 79.1 & 79.2 & 76.3 & 85.3 & 72.4 & 81.2 & 2.3 \\
\hline Years having diabetes & $8.1(0.5)$ & $10.6(0.4)$ & $10.6(0.6)$ & $11.0(0.5)$ & $10.4(0.7)$ & $9.3(0.8)$ & $10.4(1.5)$ & - \\
\hline On oral medication, \% & 63.1 & 47.4 & 49.8 & 42.8 & 47.5 & 44.2 & 44.0 & - \\
\hline On insulin, \% & 4.2 & 23.2 & 27.1 & 20.4 & 16.0 & 15.0 & 17.0 & - \\
\hline
\end{tabular}

${ }^{*}$ At baseline and unweighted statistics.

tWeighted statistics for $\mathrm{N}=11767732$

tWeighted statistics for $\mathrm{N}=80651898$.

BMI, body mass index; DBP, diastolic blood pressure; NHANES, National Health and Nutrition Examination Survey; SBP, Systolic blood pressure. 
Table 2 Comparison of prevalence of depression between the Korean Americans ( $\geq 35$ years old) with uncontrolled type 2 diabetes (A1C $\geq 7.0 \%$ ) at baseline in our clinical study, the comparison group (Americans with the same conditions as Korean Americans), and the standard group (Americans with $\mathrm{A} 1 \mathrm{C}<5.7 \%$ and not being diagnosed of diabetes, pre-diabetes or type 1 diabetes) in the NHANES

\begin{tabular}{|c|c|c|c|c|c|c|c|c|}
\hline \multirow[b]{2}{*}{ Depressionlgroups } & \multirow{2}{*}{$\begin{array}{l}\text { Korean } \\
\text { Americanst } \\
(n=249)\end{array}$} & \multicolumn{6}{|c|}{ Comparison groupł } & \multirow{2}{*}{$\begin{array}{l}\text { Standard } \\
\text { group§ } \\
(n=6880)\end{array}$} \\
\hline & & $\begin{array}{l}\text { Total } \\
(n=1707)\end{array}$ & $\begin{array}{l}\text { Whites } \\
(n=537)\end{array}$ & $\begin{array}{l}\text { Blacks } \\
(n=574)\end{array}$ & $\begin{array}{l}\text { Mexican } \\
(n=314)\end{array}$ & $\begin{array}{l}\text { Hispanic } \\
(n=161)\end{array}$ & $\begin{array}{l}\text { Others } \\
(n=121)\end{array}$ & \\
\hline \multicolumn{9}{|l|}{ Experienced at least once a week in the past 2 weeks (\%): } \\
\hline 1. Little interest or pleasure doing things & $49.8^{\star \star \star}$ & 27.7 & 27.4 & 28.9 & 26.8 & 38.4 & 18.2 & 20.1 \\
\hline 2. Feeling down, depressed, or hopeless & $39.4^{* * *}$ & 25.5 & 23.9 & 24.7 & 32.5 & 39.0 & 19.4 & 20.6 \\
\hline 3. Trouble falling or staying asleep, or sleeping too much & $59.0^{* * *}$ & 40.9 & 42.9 & 36.2 & 35.0 & 44.1 & 44.0 & 38.6 \\
\hline 4. Feeling tired or having little energy & $70.3^{\star * *}$ & 53.9 & 58.2 & 47.2 & 45.8 & 49.8 & 54.5 & 46.5 \\
\hline 5. Poor appetite or overeating & $55.4^{\star \star *}$ & 26.4 & 27.1 & 25.9 & 21.9 & 37.0 & 19.9 & 19.7 \\
\hline $\begin{array}{l}\text { 6. Feeling bad about yourself or that you are a failure or have let } \\
\text { yourself or your family down }\end{array}$ & $28.5^{\star \star \star}$ & 18.4 & 18.0 & 17.5 & 18.1 & 29.1 & 15.8 & 16.0 \\
\hline $\begin{array}{l}\text { 7. Trouble concentrating on things, such as reading the newspaper } \\
\text { or watching television }\end{array}$ & $29.3^{\star * \star}$ & 15.9 & 15.8 & 15.8 & 12.6 & 26.9 & 12.6 & 15.6 \\
\hline $\begin{array}{l}\text { 8. Moving or speaking so slowly that other people could have } \\
\text { noticed. Or the opposite being so fidgety or restless than usual }\end{array}$ & 17.3 & 13.9 & 12.0 & 14.2 & 12.7 & 29.9 & 15.7 & 8.2 \\
\hline $\begin{array}{l}\text { 9. Thoughts that you would be better off dead, or of hurting } \\
\text { yourself }\end{array}$ & $16.9^{\star \star *}$ & 5.0 & 4.2 & 5.5 & 4.7 & 13.1 & 3.8 & 2.8 \\
\hline $\begin{array}{l}\text { 10. Have these problems made it difficult for you to do your work, } \\
\text { take care of things at home, or get along with other people? }\end{array}$ & 24.4 & 33.6 & 33.2 & 30.0 & 33.3 & 35.1 & 46.1 & 26.6 \\
\hline PHQ-9, mean (SE) & $5.1(0.3)^{\star \star \star}$ & $3.7(0.1)$ & $3.6(0.2)$ & $3.7(0.3)$ & $3.6(0.3)$ & $5.0(0.7)$ & $3.1(0.5)$ & $2.7(0.1)$ \\
\hline Status, $\chi^{2}$ statistics & $23.6^{\star \star *}$ & - & - & - & - & - & - & - \\
\hline Normal (PHQ-9<5) (\%) & 55.8 & 71.3 & 71.9 & 71.9 & 71.0 & 60.2 & 74.3 & 79.9 \\
\hline Mild (PHQ-9: 5-9) (\%) & 29.3 & 17.2 & 16.9 & 16.6 & 18.0 & 20.1 & 17.5 & 13.8 \\
\hline Clinical (PHQ-9 $\geq 10)(\%)$ & 14.9 & 11.5 & 11.2 & 11.5 & 11.0 & 19.7 & 8.2 & 6.3 \\
\hline
\end{tabular}

${ }_{* \star *} p<0.001$ for unweighted test statistics compared with the comparison group total.

†At baseline.

$\ddagger \mathrm{N}=11767732$.

$\S \mathrm{N}=80651898$

IRecoded as 0 if the score was 0 , and 1 otherwise.

NHANES, National Health and Nutrition Examination Survey; PHQ-9, Patient Health Questionnaire-9. 
statistical significance varied across groups. In general, men, older people, and people with greater income and education were less likely to experience depression. For example, the effect on depression of being man was significantly lower in all groups: $\mathrm{OR}=0.67,95 \% \mathrm{CI} 0.47$ to 0.95 in KAs; OR $=0.62$, $95 \%$ CI 0.39 to 0.97 in the comparison group; and $\mathrm{OR}=0.55,95 \%$ CI 0.48 to 0.63 in the standard group. Depression decreased with age, but it was statistically significant in the standard group ( $\mathrm{OR}=0.82,95 \%$ CI 0.77 to 0.88 ).

Second, people living alone experienced greater depression, which was consistent for all groups and statistically significant for the KAs and the comparison group. KAs living alone were almost three times more likely to be depressed ( $\mathrm{OR}=2.92,95 \%$ CI 1.65 to 5.18 ) than KAs who were living with someone. The effect of living alone on depression was also statistically significant in the standard group ( $\mathrm{OR}=1.45$, 95\% CI 1.20 to 1.76$)$. Also, oral medication and insulin for DM increased the likelihood of depression in KAs, but only oral medication was statistically significant $(\mathrm{OR}=1.49,95 \%$ CI 1.07 to 2.09$)$. DM medication was, however, inversely related to depression in the comparison group, although the relationship was not statistically significant.

Third, the A1C level was significantly related to depression in the standard group $(\mathrm{OR}=1.45,95 \% \mathrm{CI}$ 1.20 to 1.76$)$, and so was the high triglyceride level (OR=1.45, 95\% CI 1.15 to 1.83$)$. Other physiological indicators (high cholesterol and high blood pressure) were not significantly related to depression.

Finally, self-efficacy for DM was correlated with depression. KAs in the middle and upper tertiles for selfefficacy were less likely to have depression $(\mathrm{OR}=0.57$, $95 \%$ CI 0.40 to 0.82 , and $\mathrm{OR}=0.45,95 \%$ CI 0.30 to 0.66 , respectively) than were KAs in the first tertile. Social support from family members and friends was significant, but the effect disappeared when the social support gap was introduced into the model. Those with a greater gap between expected and received support were one and a half times more likely to have depression $(\mathrm{OR}=1.54,95 \%$ CI 1.06 to 2.23) (see table 3$)$.

\section{Improvement of depression}

The mean depression score for KAs who completed the self-help DM intervention in the clinical trial $(n=209)$ decreased over time from $5.3(\mathrm{SE}=0.4)$ at baseline to 4.7 $(\mathrm{SE}=0.3)$ at month $3 ; 4.6(\mathrm{SE}=0.3)$ at month 6 ; and 4.4 $(\mathrm{SE}=0.3)$ at 12 months. The changes from baseline were all statistically significant at $p<0.05$. Mean changes for the intervention group from baseline to months 3,6 , and 12 were $-1.2 \quad(\mathrm{SE}=0.3),-0.8 \quad(\mathrm{SE}=0.4)$, and -1.3 $(\mathrm{SE}=0.4)$, respectively; all changes in the intervention group were statistically significant, but the changes in the control groups, which were $-0.1 \quad(\mathrm{SE}=0.4),-0.7$ $(\mathrm{SE}=0.4)$, and $-0.5(\mathrm{SE}=0.5)$ for the same period, were not significant (table 4). Similarly, of the KAs who completed the intervention, the proportion with clinical depression decreased from $16.3 \%$ at baseline to $10.7 \%$,
$13.6 \%$, and $8.1 \%$ at months 3,6 , and 12 , respectively. These changes were statistically significant $\left(\chi^{2}(6)=13.81\right.$, $\mathrm{p}=0.03) \quad($ table 5).

\section{DISCUSSION}

The findings of this research confirm our hypothesis that KA adults with DM would report a much higher rate of depressive symptoms $(44.2 \%)$ than would Americans with DM $(28.7 \%)$ or without DM $(20.1 \%)$. It was clear that the net effect of diabetes on depression was significant across all groups. For example, the prevalence of mild depression among Americans with DM was $3.4 \%$ higher than in their counterparts without DM, and the difference was statistically significant $(z=3.57,95 \%$ CI 0.014 to $0.054, \mathrm{p}<0.001)$. The prevalence of clinical depression among Americans with DM (11.5\%) was almost twice that of their counterparts without DM $(6.3 \%)$, and the difference was also significant $(\mathrm{z}=7.38$, $95 \%$ CI 0.036 to $0.068, \mathrm{p}<0.001$ ) (table 2 ).

A similar pattern was observed among KAs, although it was not feasible to directly deduce the net effect of diabetes on depression (note: no causality assumed) among KAs. Nevertheless, it is reasonable to assume that the effect was much greater among KAs than among Americans with DM. Considering the similarities in diabetes-related biomarkers (eg, A1C and total cholesterol) between KAs and Americans with DM, the net effect of diabetes on depression would have been same for both groups. Then the differences between the two groups in mild $(12.1 \%$; $\mathrm{z}=4.59,95 \%$ CI 0.062 to 0.180 , $\mathrm{p}<0.001)$ and clinical $(3.4 \% ; \mathrm{z}=1.55,95 \%$ CI -0.013 to $0.081, \mathrm{p}=0.121$ ) depression can be attributed to personal, social, and environmental factors, some of which are modifiable. In fact, KAs' depression prevalence (measured by the PHQ-9) was higher than any other previous depression estimations among KAs including those in our own studies with KA elderly ( $\geq 60$ years old, $30.3 \%)^{13}$ and South Korean elderly $(27.8 \%)^{21}$ as well as in a comparative epidemiological study of Korean, Japanese, and Chinese American adults $\left(33.3 \%, 15.7 \%\right.$, and $20.4 \%$, respectively). ${ }^{4}$ The high prevalence of depression among KAs with DM is alarming. These comorbid conditions impose substantial management burdens for this vulnerable group, who lack culturally and linguistically relevant interventions necessitated by language barriers and low health literacy. To make matters worse, KAs demonstrated significantly lower access to care than did the general population; nearly half of the participants (48\%) reported that they had no medical insurance, whereas the uninsured rates for the comparison and standard groups were $\sim 15 \%$. Given that almost a half $(44.2 \%)$ of participants with uncontrolled DM (A1C $\geq 7 \%$ ) had comorbid depression, this access gap highlights the magnitude of barriers to obtaining an optimal care among KAs with DM. Inadequate usage of healthcare services, including a lack of, or insufficient access to, 
Table 3 Comparison of people 35 years or older between Korean Americans ( $\geq 35$ years old) with uncontrolled type 2 diabetes ( $\mathrm{A} 1 \mathrm{C} \geq 7.0 \%$ at baseline) in our clinical study, and the comparison group (Americans with the same conditions as Korean Americans) and the standard group (Americans with A1C $<5.7 \%$ and not being diagnosed of diabetes, pre-diabetes or type 1 diabetes) in the NHANES, using ordered logistic regressions on depression (normal=0, mild depression=1, and clinical depression $=2$ ) on listwise deletion of missing cases

\begin{tabular}{|c|c|c|c|}
\hline Group & Korean Americanst $(n=759)$ & Comparison group $\neq(n=1000)$ & Standard group§ $(n=6185)$ \\
\hline \multirow[t]{2}{*}{ Indicators\model summary } & $\begin{array}{l}\operatorname{LR} \chi 2(20)=93.18 \\
\mathrm{p}<0.001\end{array}$ & $\begin{array}{l}F(19,45)=3.25 \\
p<0.001\end{array}$ & $\begin{array}{l}F(17,48)=19.36 \\
p<0.001\end{array}$ \\
\hline & Pseudo $R^{2}=6.8 \%$ & & \\
\hline Sex $($ female $=0$, male $=1)$ & $0.67(0.47,0.95)^{\star}$ & $0.62(0.39,0.97)^{\star}$ & $0.55(0.48,0.63)^{\star \star *}$ \\
\hline Age (in 10s) & $0.91(0.76,1.10)$ & $0.91(0.78,1.06)$ & $0.82(0.77,0.88)^{\star \star *}$ \\
\hline \multicolumn{4}{|l|}{ Income level (vs FPL $<=138 \%$ ) } \\
\hline $139-250 \%$ & $0.54(0.37,0.80)^{\star \star}$ & $0.69(0.41,1.15)$ & $0.56(0.45,0.71)^{\star * *}$ \\
\hline $251-400 \%$ & $0.80(0.52,1.25)$ & $0.69(0.40,1.20)$ & $0.41(0.33,0.52)^{\star \star \star}$ \\
\hline$>400 \%$ & $0.45(0.28,0.73)^{\star \star}$ & $0.38(0.22,0.65)^{\star \star *}$ & $0.27(0.21,0.36)^{\star \star *}$ \\
\hline \multicolumn{4}{|c|}{ Education level (vs 0-11th grade) } \\
\hline High school graduate & $0.97(0.59,1.61)$ & $0.77(0.45,1.34)$ & $0.97(0.77,1.21)$ \\
\hline College graduate or above & $1.04(0.62,1.75)$ & $0.70(0.43,1.17)$ & $0.85(0.68,1.06)$ \\
\hline Living alone (yes=1) & $2.92(1.65,5.18)^{\star \star \star}$ & $1.64(0.99,2.70)$ & $1.45(1.20,1.76)^{\star \star \star}$ \\
\hline Hemoglobin A1C (\%) & $1.02(0.93,1.13)$ & $1.06(0.93,1.20)$ & $0.76(0.58,1.00)^{\star}$ \\
\hline \multicolumn{4}{|l|}{ Triglycerides (vs <200 mg/dL) } \\
\hline Borderline $(200-239 \mathrm{mg} / \mathrm{dL})$ & $1.01(0.68,1.49)$ & $0.84(0.49,1.43)$ & $1.17(0.92,1.49)$ \\
\hline High ( $\geq 240$ mg/dL) & $1.34(0.92,1.97)$ & $1.34(0.88,2.05)$ & $1.45(1.15,1.83)^{\star \star}$ \\
\hline \multicolumn{4}{|l|}{ Obesity (vs BMI <25) } \\
\hline Overweight (BMI: 25-30) & $0.95(0.69,1.30)$ & $0.63(0.31,1.29)$ & $0.95(0.78,1.16)$ \\
\hline Obese $(\mathrm{BMI}>30)$ & $0.66(0.33,1.32)$ & $1.11(0.55,2.26)$ & $1.27(0.99,1.63)$ \\
\hline DM oral medication (yes=1) & $1.49(1.07,2.09)^{\star}$ & $0.92(0.65,1.30)$ & - \\
\hline DM insulin (yes=1) & $1.72(0.77,3.82)$ & $0.80(0.53,1.21)$ & - \\
\hline \multicolumn{4}{|l|}{ Self-efficacy (1st tertile <34\%) } \\
\hline Middle tertile (34-66\%) & $0.57(0.40,0.82)^{\star *}$ & - & - \\
\hline High tertile $(67-100 \%)$ & $0.45(0.30,0.66)^{\star \star \star}$ & - & - \\
\hline \multicolumn{4}{|c|}{ Social support gap (1st tertile $<34 \%$ ) } \\
\hline Middle tertile (34-66\%) & $1.01(0.70,1.47)$ & - & - \\
\hline High tertile $(67-100 \%)$ & $1.54(1.06,2.23)^{\star}$ & - & - \\
\hline
\end{tabular}

${ }^{*} \mathrm{p}<0.05,{ }^{* *} \mathrm{p}<0.01,{ }^{* * *} \mathrm{p}<0.001$.

†Pooled sample at baseline, months 3,6 , and 12 .

$\mp \mathrm{N}=7017657$.

$\S \mathrm{N}=74386033$; health insurance coverage, total cholesterol status, blood pressure control status, and social support were not reported due to non-significance.

BMI, body mass index; DM, diabetes mellitus; FPL, federal poverty line; NHANES, National Health and Nutrition Examination Survey.

Table 4 Depression score changes over time in the intervention and control groups in the clinical study (completer only, $\mathrm{n}=209$ )

\begin{tabular}{llcrc}
\hline Group & Baseline & Month 3 & Month 6 & Month 12 \\
\hline Intervention, mean (SE) & $5.4(0.5)$ & $4.2(0.4)$ & $4.6(0.5)$ & $4.1(0.3)$ \\
Change from baseline & - & $22121.2(0.3)^{\star \star \star}$ & $-0.8(0.4)^{\star}$ & $-1.3(0.4)^{\star \star}$ \\
Control & $5.3(0.5)$ & $5.2(0.5)$ & $4.6(0.4)$ & $4.8(0.5)$ \\
Change from baseline & & $-0.1(0.4)$ & $-0.7(0.4)$ & $-0.5(0.5)$ \\
Total & $5.3(0.4)$ & $4.7(0.3)$ & $4.6(0.3)$ & $4.4(0.3)$ \\
Change from baseline & & $-0.6(0.3)^{\star \star}$ & $-0.8(0.3)^{\star \star}$ & $-0.9(0.3)^{\star *}$ \\
\hline
\end{tabular}

${ }^{*} \mathrm{p}<0.05,{ }^{* *} \mathrm{p}<0.01,{ }^{* * *} \mathrm{p}<0.001$.

care among KAs, is well documented. ${ }^{22}{ }^{23}$ One explanation for this is that a large proportion of KAs work in or own small businesses that cannot afford medical insurance. Also, some recent KA immigrants over age 65 cannot enroll in Medicare because they lack an adequate employment history.
Several researchers have argued that KAs and other Asian American immigrants are susceptible to depression because of their social and linguistic isolation and because of the insufficient access to care suffered by first-generation immigrants. ${ }^{13} 24$ Our findings show that those who have expected more social support than they 
Table 5 Change in distribution of depression over time in the clinical study (completer only, $\mathrm{n}=209)^{\star}$

\begin{tabular}{|c|c|c|c|c|c|}
\hline Depression level & Baseline (\%) & Month $3(\%)$ & Month 6 (\%) & Month 12 (\%) & Total (\%) \\
\hline \multicolumn{6}{|l|}{ Intervention group } \\
\hline Normal & 53.3 & 61.9 & 68.6 & 68.6 & 63.1 \\
\hline Mild depression & 28.6 & 29.5 & 21.8 & 22.9 & 24.5 \\
\hline Clinical depression & 18.1 & 8.6 & 13.6 & 8.6 & 12.4 \\
\hline \multicolumn{6}{|l|}{ Control group } \\
\hline Normal & 53.9 & 51.0 & 60.4 & 52.9 & 54.5 \\
\hline Mild depression & 31.7 & 36.0 & 26.7 & 39.4 & 33.5 \\
\hline Clinical depression & 14.4 & 13.0 & 12.8 & 7.7 & 12.0 \\
\hline \multicolumn{6}{|l|}{ Total } \\
\hline Normal & 53.6 & 56.6 & 64.6 & 60.8 & 58.9 \\
\hline Mild depression & 30.1 & 32.7 & 21.8 & 31.1 & 30.0 \\
\hline Clinical depression & 16.3 & 10.7 & 13.6 & 8.1 & 12.2 \\
\hline
\end{tabular}

ultimately receive are more likely to report depressive symptoms.

A unique contextual factor associated with the high prevalence of depression among new immigrants may stem from an incongruity between educational attainment and both income and occupational prestige. The higher educational achievement among the firstgeneration KAs does not translate into professional jobs or high incomes in the USA. The incongruity between high aspirations before immigration and actual attainment after immigration quantified using socioeconomic status has been identified as a risk factor for poor mental health. ${ }^{25}$ The dissonance between expected and attained socioeconomic status can further exacerbate KAs' mental health via insufficient English skills and low health literacy. Many KAs have described their struggles in communicating with medical providers as well as a lack of access to health information in their mother tongue. ${ }^{26}$ In some KA households, children act as translators or brokers between their immigrant parents and healthcare providers as well as the mainstream culture. The reversed roles between parents and children can pose mental health challenges for some KAs because they compromise parents' cultural positions or status as 'heads of household' or 'village leaders', roles that are traditionally assured and reserved for parents in Korean and other Asian cultures. ${ }^{13}$ Thus, contextual factors related to acculturation and subsequent acculturative stress may explain the gaps in the prevalence of depression between KAs and other groups. In addition, unlike in other population literature, 'marriage', 'living with family member', and 'high education level' are not always good proxies for social support or personal resources that mediate stress levels. Regardless of the level of social support received from family members, most KAs feel that they are linguistically isolated from the larger mainstream community. Despite the high education level that many have obtained in their motherland, most KA immigrants suffer from high levels of English insufficiency and low health literacy, which also lead to inadequate healthcare access and information.
Our findings are also consistent with previous research showing significant correlates of depressive symptoms: being woman, younger, in a lower income level, less educated, and living alone emerged as statistically significant correlates in all three groups (KAs with DM, Americans with DM, Americans without DM). Unique to the KAs with DM, taking DM medication was a significant correlate of depression. However, we are not certain that there is an etiological link between taking DM medication and depressive symptoms; it is plausible that taking medication might be a proxy for the severity of DM, because many KAs do not seek DM treatment until after their disease is advanced, owing to their limited access to care.

The improvement in depressive symptoms over time differed between the intervention group, which received a self-help behavioral intervention, and the control group, which participated in data collection only. Although both groups improved in alleviating depressive symptoms during the project period (3, 6, and 12 months after the initial behavioral intervention), the intervention group's improvement was significant $(>20 \%$ reduction in depressive symptoms).

The intervention group and the control group showed statistically and clinically significant differences in glucose control: the intervention group achieved $>1.5 \%$ sustained hemoglobin A1C reduction over 12 months after the initial intervention. The premise of the selfhelp intervention was to empower these individuals by cultivating their self-efficacy and their capabilities to manage their DM condition. ${ }^{14} 15$ Although the intervention was not specifically designed to improve mental health, the participants' improved problem-solving skills and self-confidence may yet have had a therapeutic effect.

Further, our study demonstrates that depressive symptoms can be mitigated by enhancing one's coping skills, including self-efficacy, one's level of health literacy, and glucose control. To the best of our knowledge, our clinical trial was the first to target this understudied population by aiming to improve physical and mental health among KAs with uncontrolled DM for 12 months. Our 
findings demonstrate that a culturally tailored, community-based self-help intervention was effective for managing DM and depression in an ethnic/linguistic minority community. Although the sample was limited to one ethnic minority group, the study provides unique insights into the relationship between the most common comorbid conditions, DM and depression, as well as potential strategies to tackle such clinical challenges for similarly vulnerable populations.

\section{LIMITATIONS}

This study was carried out at a single site centered in the Baltimore-Washington area, targeting KAs only. We overcame this limitation by comparative analysis with a national sample that used the same measurements. Another inherent limitation is that the study's data were obtained from a DM intervention trial and did not include data from KAs without DM. Future studies should include such a comparison group to validate our findings.

Acknowledgements The authors are grateful for substantial editorial support with manuscript development provided by Dr John Bellquist at the Cain Center for Nursing Research and the Center for Transdisciplinary Collaborative Research in Self-Management Science (P30, NR015335) at The University of Texas at Austin School of Nursing.

Contributors MTK and KBK had full access to the data and drafted the manuscript. JK researched data and YJ, HBL, and DL discussed/edited the manuscript.

Funding This study was supported by a grant from the National Institute of Diabetes and Digestive and Kidney Diseases (R18DK083936) and with material support from LifeScan, including devices (OneTouch glucometers, OneTouch UltraSoft test strips, and OneTouch UltraSoft Lancets) for study participants. Also, the Johns Hopkins Institute for Clinical and Translational Research supported the cost of blood serum laboratory tests.

Disclaimer The content is solely the responsibility of the authors and does not necessarily represent the official views of the National Institutes of Health or other supporters.

Competing interests None declared.

Patient consent Obtained.

Ethics approval The study received ethics approval by Johns Hopkins Medicine IRB.

Provenance and peer review Not commissioned; externally peer reviewed.

Data sharing statement We shall make data available to the scientific community as few restrictions as feasible. However, because of the small sample size from a single ethnic community and some qualitative data (in Korean), PIs will maintain the electronic data file by removing all identifiable information. Data include demographic information, diabetes control status, and other standard blood serum test results. Further information can be obtained from the corresponding author.

Open Access This is an Open Access article distributed in accordance with the Creative Commons Attribution Non Commercial (CC BY-NC 4.0) license, which permits others to distribute, remix, adapt, build upon this work noncommercially, and license their derivative works on different terms, provided the original work is properly cited and the use is non-commercial. See: http:// creativecommons.org/licenses/by-nc/4.0/

\section{REFERENCES}

1. Anderson RJ, Freedland KE, Clouse RE, et al. The prevalence of comorbid depression in adults with diabetes: a meta-analysis. Diabetes Care 2001;24:1069-78.
2. Bogner HR, Morales KH, Post EP, et al. Diabetes, depression, and death: a randomized controlled trial of a depression treatment program for older adults based in primary care (PROSPECT). Diabetes Care 2007;30:3005-10.

3. Holt RIG, de Groot M, Golden SH. Diabetes and depression. Curr Diab Rep 2014;14:491.

4. Kim HJ, Park E, Storr CL, et al. Depression among Asian-American adults in the community: systematic review and meta-analysis. PLoS ONE 2015;10:e0127760.

5. Holt RIG, Katon WJ. Dialogue on diabetes and depression: dealing with the double burden of co-morbidity. J Affect Disord 2012;142: S1-3.

6. Ell K, Aranda MP, Xie B, et al. Collaborative depression treatment in older and younger adults with physical illness: pooled comparative analysis of three randomized clinical trials. Am J Geriatr Psychiatry 2010;18:520-30.

7. Ell K, Katon W, Lee $\mathrm{P}$, et al. Depressive symptom deterioration among predominantly Hispanic diabetes patients in safety net care. Psychosomatics 2012;53:347-55.

8. Fuentes D, Aranda MP. Depression interventions among racial and ethnic minority older adults: a systematic review across 20 years. Am J Geriatr Psychiatry 2012;20:915-31.

9. Ockene IS, Tellez TL, Rosal MC, et al. Outcomes of a Latino community-based intervention for the prevention of diabetes: the Lawrence Latino Diabetes Prevention Project. Am J Public Health 2012;102:336-42.

10. Bernstein KS, Park S, Shin J, et al. Acculturation, discrimination and depressive symptoms among Korean immigrants in New York City. Community Ment Health J 2011;47:24-34.

11. Park SY, Cho S, Park Y, et al. Factors associated with mental health service utilization among Korean American immigrants. Community Ment Health J 2013;49:765-73.

12. Pistulka GM, Winch PJ, Park $\mathrm{H}$, et al. Maintaining an outward image: a Korean immigrant's life with type 2 diabetes mellitus and hypertension. Qual Health Res 2012;22:825-34.

13. Kim MT, Kim KB, Han HR, et al. Prevalence and predictors of depression in Korean American elderly: findings from the memory and aging study of Koreans (MASK). Am J Geriatr Psychiatry 2015;23:671-83.

14. Kim KB, Kim MT, Lee HB, et al. Community health workers versus nurses as counselors or case managers in a self-help diabetes management program. Am J Public Health 2016;106:1052-8

15. Kim MT, Kim KB, Huh B, et al. The effect of a community-based self-help intervention: Korean Americans with type 2 diabetes. $A m$ J Prev Med 2015;49:726-37.

16. Han C, Jo SA, Kwak JH, et al. Validation of the Patient Health Questionnaire-9 Korean version in the elderly population: the Ansan Geriatric Study. Compr Psychiatry 2008;49:218-23.

17. Shin J, Park S, Cho S, et al. Validation of Patient Health Questionnaire-9 Korean Version (PHQ-9K) Scale for screening depression among Korean Americans in community settings. $J$ Theory Constr Test 2010;14:45-51.

18. Lorig KR, Sobel DS, Ritter PL, et al. Effect of a self-management program on patients with chronic disease. Eff Clin Pract 2001;4:256-62.

19. Han H, Kim MT, Weinert C. The psychometric evaluation of Korean translation of the Personal Resource Questionnaire 85-Part 2. Nurs Res 2002;51:309-16.

20. Weinert C. A social support measure: PRQ85. Nurs Res 1987;36:273-7.

21. Park JH, Kim KW, Kim MH, et al. A nationwide survey on the prevalence and risk factors of late life depression in South Korea. $J$ Affect Disord 2012;138:34-40.

22. Han H, Kim J, Kim MT, et al. Measuring health literacy among immigrants with a phonetic primary language: a case of Korean American women. J Immig Minor Health 2011;13:253-9.

23. Kandula NR, Kersey M, Lurie N. Assuring the health of immigrants: what the leading health indicators tell us. Annu Rev Public Health 2004;25:357-76.

24. Mitra M, lezzoni LI, Zhang J, et al. Prevalence and risk factors for postpartum depression symptoms among women with disabilities. Matern Child Health J 2015;19:362-72.

25. Sorensen MV, Snodgrass JJ, Leonard WR, et al. Lifestyle incongruity, stress and immune function in indigenous Siberians: the health impacts of rapid social and economic change. Am J Phys Anthropol 2009;138:62-9.

26. Han H, Kang J, Kim KB, et al. Barriers to and strategies for recruiting Korean Americans for community-partnered health promotion research. J Immigr Minor Health 2007;9:137-46. 